\title{
Chaotic Dynamics in a Flexible Exchange Rate System: A Study of Noise Effects
}

\author{
TOICHIRO ASADA ${ }^{\mathrm{a}, *}$, TETSUYA MISAWA $^{\mathrm{b}, \dagger}$ and TOSHIO INABA ${ }^{\mathrm{c}, \uparrow}$ \\ ${ }^{a}$ Faculty of Economics, Chuo University, 742-1, Higashinakano, Hachioji, Tokyo 192-0393, Japan; \\ ${ }^{\mathrm{b}}$ Faculty of Economics, Nagoya City University, Mizuho-cho, Mizuho-ku, Nagoya 467-0001, Japan; ${ }^{\mathrm{c}}$ School of Education, \\ Wasada University, 1-6-1, Nishiwaseda, Shinjuku-ku, Tokyo 169-0051, Japan
}

(Received 18 August 1999)

\begin{abstract}
In this paper, we investigate by means of analytical method and numerical simulations the properties of three-dimensional business cycle model, in which foreign exchange rate is flexible and a parameter is fluctuated by noise. The model is a discrete time version of Asada (Journal of Economics, 62, 239-269, 1995)'s continuous time open economy model without noise. We show (1) noise may suppress the burst of flexible foreign exchange rate when its behavior begins to burst as a bifurcation parameter (adjustment speed of the goods market) is increased, (2) the windows of cycles can be broken by noise, and (3) noise may reveal the hidden structures.
\end{abstract}

Keywords: Chaotic dynamics, Flexible exchange rates, Noise effects, Small open economy

\section{INTRODUCTION}

In general, an economy is not an isolated system but it is subject to the disturbances from other subsystems of the society. This observation is particularly important for theoretical and empirical investigations in international economics or regional sciences, which study the economic interactions between several regions. Asada (1995) presented a dynamic model of small open economy by introducing international trade and international capital movement into the Kaldorian business cycle theory.
Asada (1995) investigated both of the fixed exchange rate system and the flexible exchange rate system in a framework of the continuous time model without stochastic disturbance (noise). Asada et al. (1998) studied a discrete time version of the fixed exchange rate system with noise effects by means of numerical simulations, and showed that such a system can produce very complex behavior including chaos.

This paper also considers a discrete time version of the Kaldorian business cycle model in a small open economy with noise effects, but in this study

\footnotetext{
* Corresponding author. E-mail: asada@tamacc.chuo-u.ac.jp

† E-mail:misawa@econ.nagoya-cu.ac.jp

‡ E-mail:inaba@mn.waseda.ac.jp
} 
we concentrate on the system of flexible exchange rates. We investigate by means of analytical method and numerical simulations the properties of threedimensional system, in which a parameter which represents the adjustment speed of the adaptive expectation of exchange rate is fluctuated by noise. Asada et al. (1998) showed in a framework of the fixed exchange rate system that the noise may not only obscure the underlying structures, but also reveal the hidden structures, for example, chaotic attractors near the window. In this paper, we show (1) noise may suppress the burst of flexible exchange rate when its behavior begins to burst as a bifurcation parameter (adjustment speed of the goods market) is increased, (2) the windows of cycle can be broken by noise, and (3) noise may reveal the hidden structures.

\section{FORMULATION OF THE MODEL}

The basic system of equations is given as follows*:

$$
\begin{gathered}
Y_{t+1}-Y_{t}=\alpha\left[C_{t}+I_{t}+G+J_{t}-Y_{t}\right] ; \quad \alpha>0 \\
K_{t+1}-K_{t}=I_{t} \\
C_{t}=c\left(Y_{t}-T_{t}\right)+C_{0} ; \quad 0<c<1, C_{0}>0, \\
I_{t}=I\left(Y_{t}, K_{t}, r_{t}\right) ; \quad I_{Y} \equiv \partial I_{t} / \partial Y_{t}>0 \\
I_{K} \equiv \partial I_{t} / \partial K_{t}<0, \quad I_{t} \equiv \partial I_{t} / \partial r_{t}<0 \\
T_{t}=\tau Y_{t}-T_{0} ; \quad 0<\tau<1, T_{0}>0 \\
M_{t} / p=L\left(Y_{t}, r_{t}\right) ; \quad L_{Y} \equiv \partial L_{t} / \partial Y_{t}>0 \\
L_{t} \equiv \partial L_{t} / \partial r_{t}<0, \\
J_{t}=J\left(Y_{t}, E_{t}\right) ; \quad J_{Y} \equiv \partial J_{t} / \partial Y_{t}<0 \\
J_{E} \equiv \partial J_{t} / \partial E_{t}>0 \\
Q_{t}=\beta\left\{r_{t}-r_{\mathrm{f}}-\left(E_{t}^{\mathrm{e}}-E_{t}\right) / E_{t}\right\} ; \quad \beta>0
\end{gathered}
$$

$$
\begin{gathered}
A_{t}=J_{t}+Q_{t}, \\
A_{t}=0, \\
E_{t+1}^{\mathrm{e}}-E_{t}^{\mathrm{e}}=\left(\gamma+\sigma \varepsilon_{t}\right)\left(E_{t}-E_{t}^{\mathrm{e}}\right) ; \quad \gamma>0, \sigma \geqq 0, \\
M_{t}=\bar{M},
\end{gathered}
$$

where the meanings of the symbols are as follows. $Y=$ net real national income, $C=$ real consumption expenditure, $I=$ net real private investment expenditure, $G=$ real government expenditure (fixed), $K=$ real physical capital stock, $T=$ real income tax, $M=$ nominal money supply, $p=$ price level (fixed), $r=$ nominal domestic rate of interest, $r_{\mathrm{f}}=$ nominal foreign rate of interest (fixed), $E=$ value of a unit of foreign currency in terms of domestic currency (exchange rate), $E^{\mathrm{e}}=$ expected exchange rate of near future, $J=$ balance of current account (net export) in real terms, $Q=$ balance of capital account in real terms, $A=$ total balance of payments in real terms, $\alpha=$ adjustment speed in the goods market, $\beta=$ parameter which represents the 'degree of capital mobility', $\gamma=$ parameter which represents the "speed of adaptation" of the expected exchange rate, $\varepsilon=$ normal pseudo random number $N(0,1), \sigma=$ standard deviation parameter. The subscript $t$ denotes time period.

Equation (1) represents the quantity adjustment process in the goods market. Equation (2) says that the physical capital stock increases or decreases according as the net investment is positive or negative. Equations (3), (4), and (5) are consumption function, investment function, and income tax function respectively. Equation (6) is the equilibrium condition for the money market. Equation (7) says that the current account is determined by $Y_{t}$ and $E_{t}$, which is a standard type of the current account function. Equation (8) formalizes the idea that the capital account becomes positive or negative according as the difference between the rates of return of domestic and foreign bonds is positive or negative. We can consider $\beta$ as the index of the degree of the capital mobility. Equation (9) is

* Equations (1)-(7) in this paper are identical to those in a fixed exchange rate system which was presented in Asada et al. (1998). 
the definition of the total balance of payments. Equation (10) is a characterization of the flexible exchange rate system, i.e., it is assumed that the exchange rate is adjusted instantaneously to keep the equilibrium of the total balance of payments $\left(A_{t}=0\right)$. Equation (11) is a formalization of the 'adaptive expectation hypothesis' concerning the expected exchange rate. It is assumed that the speed of adaptation is fluctuated by noise. Equation (12) says that under flexible exchange rate system the domestic monetary authority can control money supply contrary to the case of fixed exchange rate system, so that we can consider the money supply $(M)$ as an exogenous variable. ${ }^{\dagger}$

We can reduce the above system (1)-(12) to the following system of equations ${ }^{\ddagger}$ :

(i) $Y_{t+1}-Y_{t}=\alpha\left[(1-\tau) Y_{t}+c T_{0}+C_{0}\right.$

$$
\begin{aligned}
& +G+I\left(Y_{t}, K_{t}, r\left(Y_{t}, \bar{M}\right)\right) \\
& \left.+J\left(Y_{t}, E_{t}\right)-Y_{t}\right] ; \quad \alpha>0,
\end{aligned}
$$

(ii) $K_{t+1}-K_{t}=I\left(Y_{t}, K_{t}, r\left(Y_{t}, \bar{M}\right)\right)$,

(iii) $A_{t}=J\left(Y_{t}, E_{t}\right)+\beta\left\{r\left(Y_{t}, \bar{M}\right)\right.$

$$
\left.-r_{\mathrm{f}}-E_{t}^{\mathrm{e}} / E_{t}+1\right\}=0,
$$

(iv) $E_{t+1}^{\mathrm{e}}-E_{t}^{\mathrm{e}}=\left(\gamma+\sigma \varepsilon_{t}\right)\left(E_{t}-E_{t}^{\mathrm{e}}\right)$.

Solving Eq. (13)(iii) with respect to $E_{t}$, we obtain

$$
\begin{aligned}
E_{t} & =E\left(Y_{t}, E_{t}^{\mathrm{e}} ; \beta\right), \\
E_{Y} & \equiv \partial E_{t} / \partial Y_{t}=\left(-J_{Y}-\beta r_{Y}\right) /\left(J_{E}+\beta E_{t}^{\mathrm{e}} / E_{t}^{2}\right) \\
& =\left(m-\beta r_{Y}\right) /\left(J_{E}+\beta E_{t}^{\mathrm{e}} / E_{t}^{2}\right) \\
& \gtreqless 0 \Longleftrightarrow \beta \lesseqgtr m / r_{Y}, \\
E_{E}^{\mathrm{e}} & =\partial E_{t} / \partial E_{t}^{\mathrm{e}}=\beta /\left(J_{E} E_{t}+\beta E_{t}^{\mathrm{e}} / E_{t}\right)>0,
\end{aligned}
$$

where $m \equiv-J_{Y} \equiv-\partial J_{t} / \partial Y_{t}>0, \quad r_{Y} \equiv \partial r_{t} / \partial Y_{t}>0$, and $J_{E} \equiv \partial J_{t} / \partial E_{t}>0$. Equation (14) implies that $E_{t}$ is an increasing function of $Y_{t}$ when the degree of capital mobility $(\beta)$ is sufficiently small, but it becomes a decreasing function of $Y_{t}$ when $\beta$ is sufficiently large. $\S$

Substituting Eq. (14) into (13), we obtain the following system of 'fundamental dynamical equations':

(i) $Y_{t+1}=Y_{t}+\alpha\left[(1-\tau) Y_{t}+c T_{0}\right.$

$$
\begin{aligned}
& +C_{0}+G+I\left(Y_{t}, K_{t}, r\left(Y_{t}, \bar{M}\right)\right) \\
& \left.+J\left(Y_{t}, E\left(Y_{t}, E_{t}^{\mathrm{e}} ; \beta\right)\right)-Y_{t}\right] \\
\equiv & F_{1}\left(Y_{t}, K_{t}, E_{t}^{\mathrm{e}} ; \alpha, \beta\right),
\end{aligned}
$$

(ii) $K_{t+1}=K_{t}+I\left(Y_{t}, K_{t}, r\left(Y_{t}, \bar{M}\right)\right) \equiv F_{2}\left(Y_{t}, K_{t}\right)$,

(iii) $E_{t+1}^{\mathrm{e}}=E_{t}^{\mathrm{e}}+\left(\gamma+\sigma \varepsilon_{t}\right)\left\{E\left(Y_{t}, E_{t}^{\mathrm{e}} ; \beta\right)-E_{t}^{\mathrm{e}}\right\}$

$$
\equiv F_{3}\left(Y_{t}, E_{t}^{\mathrm{e}} ; \beta, \gamma, \sigma\right) \text {, }
$$

On the other hand, the continuous time version without noise effect which was formulated in Asada (1995) is read as

(i) $\mathrm{d} Y / \mathrm{d} t=\alpha\left[(1-\tau) Y+c T_{0}+C_{0}\right.$

$$
\begin{aligned}
& +G+I(Y, K, r(Y, \bar{M})) \\
& \left.+J\left(Y, E\left(Y, E^{\mathrm{e}} ; \beta\right)\right)-Y\right] \\
\equiv & f_{1}\left(Y, K, E^{\mathrm{e}} ; \alpha, \beta\right),
\end{aligned}
$$

(ii) $\mathrm{d} K / \mathrm{d} t=I(Y, K, r(Y, \bar{M})) \equiv f_{2}(Y, K)$,

(iii) $\mathrm{d} E^{\mathrm{e}} / \mathrm{d} t=\gamma\left\{E\left(Y, E^{\mathrm{e}} ; \beta\right)-E^{\mathrm{e}}\right\}$

$$
\equiv f_{3}\left(Y, E^{\mathrm{e}} ; \beta, \gamma\right) \text {. }
$$

It is easily shown that the equilibrium point $\left(Y^{*}, K^{*}, E^{\mathrm{e} *}\right)$ of the system $\left(\mathrm{S}_{1}\right)$ is identical to that of the system $\left(\mathrm{S}_{2}\right)$, and Asada (1995) showed that there exists an equilibrium point $\left(Y^{*}, K^{*}, E^{\mathrm{e} *}\right)>$ $(0,0,0)$ in the system $\left(\mathbf{S}_{2}\right)$ under some reasonable conditions. From now on, we assume that there exists an economically meaningful equilibrium point in the system $\left(\mathrm{S}_{1}\right)$.

\footnotetext{
${ }^{\dagger}$ Under the fixed exchange rate system, money supply endogenously fluctuates according as the total balance of payments is positive or negative. See Asada (1995) and Asada et al. (1998).

$\ddagger$ In Eq. (13), $r\left(Y_{t}, \bar{M}\right)$ is the 'LM equation’ which is the solution of Eq. (6) with respect to $r_{t}$.

"Solving Eq. (6) with respect to $r_{t}$, we have $r_{t}=r\left(Y_{t}, \bar{M}\right) ; r_{Y} \equiv \partial r_{t} / \partial Y_{t}=-L_{Y} / L_{r}>0$, where $L_{Y} \equiv \partial L_{t} / \partial Y_{t}>0$ and $L_{t} \equiv \partial L_{t} / \partial r_{r}<0$.

$\S$ The economic implication of this result is very clear. When $Y_{t}$ increases, the current account $\left(J_{t}\right)$ decreases through the increase of the import, while the capital account $\left(Q_{t}\right)$ increases through the increase of the domestic rate of interest. If $\beta$ is small, the "current account effect' dominates the 'capital account effect' so that the total balance of payments $\left(A_{t}\right)$ decreases. In this case, the exchange rate $\left(E_{t}\right)$ must increase to keep the equilibrium of the balance of payments $\left(A_{t}=0\right)$. On the other hand, if $\beta$ is large, the 'capital account effect' dominates so that $A_{t}$ increases. In this case, $E_{t}$ must decrease to keep $A_{t}=0$.
} 


\section{LOCAL STABILITY - INSTABILITY ANALYSIS}

Asada (1995) proved the following propositions.

(1) The equilibrium point of the system $\left(S_{2}\right)$ is locally stable if $\beta$ is sufficiently large.

(2) Suppose that $I_{Y}+I_{r} r_{Y}>1-c(1-\tau)$ at the equilibrium point. Then, the equilibrium point of the system $\left(\mathrm{S}_{2}\right)$ becomes locally unstable when $\beta$ is sufficiently small and $\alpha$ is sufficiently large.

This proposition implies that in a continuous time version of our model, large capital mobility between countries (or regions) tends to stabilize the system. Does this conclusion also apply to the discrete time version? In fact, proposition (1) does not apply to the system $\left(\mathrm{S}_{1}\right)$, because in the discrete time version the 'overshooting phenomena' are not negligible so that the system becomes unstable when the degree of capital mobility is too large. Now, let us prove this assertion formally by assuming $\sigma=0$ (no stochastic disturbance).

We can write the Jacobian matrix of the system $\left(\mathrm{S}_{1}\right)$ which is evaluated at the equilibrium point as follows:

$$
J_{1}=\left[\begin{array}{lll}
F_{11}(\alpha, \beta) & F_{12}(\alpha) & F_{13}(\alpha, \beta) \\
F_{21} & F_{22} & 0 \\
F_{31}(\beta, \gamma) & 0 & F_{33}(\beta, \gamma)
\end{array}\right],
$$

where

$$
\begin{aligned}
& F_{11}(\alpha, \beta)=1+\alpha \underset{(+)}{\alpha I_{Y}}+\underset{(-)}{I_{r}} \underset{(+)}{r_{Y}} \\
& \left.-\{1-c(1-\tau)+\underset{(+)}{m}\}+\underset{(+)}{J_{E}} E_{Y}(\beta)\right], \\
& F_{12}(\alpha)=\alpha I_{K}<0, \quad F_{13}(\alpha, \beta)=\alpha J_{E} E_{E}(\beta)>0, \\
& F_{21}=\underset{(+)}{I_{Y}}+\underset{(-) \underset{r}{I_{(+)}}}{r_{Y}}, \quad F_{22}=1+\underset{(-)}{I_{K}} \\
& F_{31}(\beta, \gamma)=\underset{(?)}{E_{Y}(\beta),} \\
& F_{33}(\beta, \gamma)=1+\gamma\left\{E_{E}(\beta)-1\right\} \\
& =1-\left(J_{E} E \gamma\right) /\left(J_{E} E+\beta\right) \text {. }
\end{aligned}
$$

We can write the characteristic equation of this system as

$$
\psi(\lambda)=\left|\lambda I-J_{1}\right|=\lambda^{3}+a_{1} \lambda^{2}+a_{2} \lambda+a_{3}=0,
$$

where

(i) $a_{1}=-$ trace $J_{1}$

$$
=-F_{11}(\alpha, \beta)-F_{22}-F_{33}(\beta, \gamma),
$$

(ii) $a_{2}=\left|\begin{array}{cc}F_{22} & 0 \\ 0 & F_{33}\end{array}\right|+\left|\begin{array}{cc}F_{11} & F_{13} \\ F_{31} & F_{33}\end{array}\right|$

$$
+\left|\begin{array}{ll}
F_{11} & F_{12} \\
F_{21} & F_{22}
\end{array}\right|
$$

$$
\begin{aligned}
= & F_{22} F_{33}(\beta, \gamma)+F_{11}(\alpha, \beta) F_{33}(\beta, \gamma) \\
& -F_{13}(\alpha, \beta) F_{31}(\beta, \gamma)+F_{11}(\alpha, \beta) F_{22} \\
& -F_{12}(\alpha, \beta) F_{21},
\end{aligned}
$$

(iii) $a_{3}=-\operatorname{det} J_{1}$

$$
\begin{aligned}
= & -F_{11}(\alpha, \beta) F_{22} F_{33}(\beta, \gamma) \\
& +F_{13}(\alpha, \beta) F_{22} F_{31}(\beta, \gamma) \\
& +F_{12}(\alpha) F_{21} F_{33}(\beta, \gamma) .
\end{aligned}
$$

It follows from the Cohn-Schur conditions for local stability that the system $\left(\mathrm{S}_{1}\right)$ is locally stable if and only if the following conditions are satisfied ${ }^{\|}$

(i) $1+a_{2}-\left|a_{1}+a_{3}\right|>0$,

(ii) $1-a_{2}+a_{1} a_{3}-a_{3}^{2}>0$,

(iii) $a_{2}<3$.

Therefore, the equilibrium point of the system $\left(\mathrm{S}_{1}\right)$ becomes locally unstable if the inequality $a_{2}>3$ is satisfied. The following proposition is a simple corollary of this fact.

\section{Proposition Suppose that}

$$
0<\underset{(+)}{I_{Y}}+\underset{(-)}{I_{r}} r_{Y}<\underbrace{1-c(1-\tau)}_{(+)}+\underset{(+)}{m}
$$

\footnotetext{
" See, for example, Gandolfo (1996) Chap. 7. In fact, the inequality (18)(iii) is redundant because this inequality can be derived from other two inequalities. Nevertheless, the inequality (18)(iii) as a necessary condition for local stability is useful for our purpose.
} 
and

$$
I_{K}<-1 \text {. }
$$

Then, the equilibrium point of the system $\left(\mathrm{S}_{1}\right)$ is locally unstable when either of the following conditions (A) or (B) is satisfied.

(A) The parameters $\beta$ and $\alpha$ are sufficiently large.

(B) The parameters $\beta$ and $\gamma$ are sufficiently large.

Proof From Eq. (14), the definitions of $F_{31}(\beta, \gamma)$ and $F_{33}(\beta, \gamma)$, the fact that $E^{\mathrm{e}}=E$ at the equilibrium point we have the following relationships:

$$
\begin{aligned}
\lim _{\beta \rightarrow \infty} J_{E} E_{Y} & =\lim _{\beta \rightarrow \infty}\left(m-\beta r_{Y}\right) /\left(1+\beta / J_{E} E\right) \\
& =-r_{Y} J_{E} E<0, \\
\lim _{\beta \rightarrow \infty} F_{31}(\beta, \gamma) & =\lim _{\beta \rightarrow \infty} \gamma\left(m-\beta r_{Y}\right) /\left(J_{E}+\beta\right) \\
& =-\gamma r_{Y}<0, \\
\lim _{\beta \rightarrow \infty} F_{33}(\beta, \gamma) & =\lim _{\beta \rightarrow \infty}\left[1+\gamma\left\{\beta /\left(J_{E} E+\beta\right)-1\right\}\right] \\
& =1 .
\end{aligned}
$$

Therefore, we obtain the following expression

$$
\begin{aligned}
A \equiv & \lim _{\beta \rightarrow \infty} a_{2}=F_{(-)} F_{(22}+F_{(-)}^{*}(\alpha) \underbrace{\left(1+F_{22}\right)}_{(-)} \\
& -F_{(+)}^{*}(\alpha) \underset{(-)}{F_{31}^{*}(\gamma)-F_{12}^{*}(-)(\alpha) F_{21},},
\end{aligned}
$$

where $F_{i j}^{*}=\lim _{\beta \rightarrow \infty} F_{i j}, \partial F_{11}^{*}(\alpha) / \partial \alpha<0, \partial F_{12}^{*}(\alpha) /$ $\partial \alpha<0, \partial F_{13}^{*}(\alpha) / \partial \alpha>0$, and $\partial F_{31}^{*}(\gamma) / \partial \gamma<0$. It follows from Eq. (22) that we have $A>3$ for sufficiently large $\beta$ and $\alpha$, or alternatively, for sufficiently large $\beta$ and $\gamma$. This proves the proposition.

This proposition shows that under some additional conditions the high degree of capital mobility $(\beta)$ combined with high adjustment speed in the goods market $(\alpha)$ or high speed of adaptation of the expected exchange rate $(\gamma)$ tends to destabilize the system $\left(S_{1}\right)$ because of the overshooting phenomena contrary to the continuous time model.

\section{NUMERICAL SIMULATIONS}

In this section, we shall present the results of some numerical simulations of the model which was formulated in the previous section. We adopt the following specifications of the functions and parameter values:

$$
I\left(Y_{t}, K_{t}, r_{t}\right)=f\left(Y_{t}\right)-0.3 K_{t}-r_{t}+100
$$

$$
\begin{gathered}
f\left(Y_{t}\right)=(80 / 3.1415) \operatorname{Arctan}\{(2.25 \times 3.1415 / 20) \\
\left.\times\left(Y_{t}-112\right)\right\}+35, \\
r_{t}=r\left(Y_{t}, M\right)=10 \sqrt{Y_{t}}-M, \\
J\left(Y_{t}, E_{t}\right)=-0.3 Y_{t}+100-100 / E_{t}, \\
E_{t+1}^{\mathrm{e}}-E_{t}^{\mathrm{e}}=\left(\gamma+\sigma \varepsilon_{t}\right)\left(E_{t}-E_{t}^{\mathrm{e}}\right), \\
\begin{array}{l}
c=0.8, \quad \tau=0.2, \quad r_{\mathrm{f}}=6, \quad c T_{0}+C_{0}+G=238, \\
M=100, \quad \beta=15, \quad \gamma=1.2 .
\end{array}
\end{gathered}
$$

Equation (24) is a formalization of the Kaldorian S-shaped investment function. Substituting Eqs. (25) and (26) into the equilibrium condition of the balance of payments (Eq. (13)(iii)), we have

$$
\begin{aligned}
& -0.3 Y_{t}+100-100 / E_{t} \\
& \quad+15\left(10 \sqrt{Y_{t}}-E_{t}^{\mathrm{e}} / E_{t}-105\right)=0 .
\end{aligned}
$$

Solving Eq. (29) with respect to $E_{t}$, we obtain the following expression for the exchange rate

$$
\begin{aligned}
E_{t}= & \left(100+15 E_{t}^{\mathrm{e}}\right) /\left(-0.3 Y_{t}\right. \\
& \left.+150 \sqrt{Y_{t}}-1475\right) .
\end{aligned}
$$

By using these data, we studied the numerical simulation. We selected the parameter $\alpha$ as a bifurcation parameter. When there is no stochastic 
disturbance $(\sigma=0)$, the equilibrium point is $\left(Y^{*}\right.$, $\left.K^{*}, E^{\mathrm{e} *}\right)=\left(Y^{*}, K^{*}, E^{*}\right) \simeq(112,450,1.56)$, which is independent of the value of the parameter $\alpha$.

In order to investigate the noise effect, the speed of adjustment in adaptive expectation hypothesis of exchange rate $(\gamma)$ is fluctuated by noise. In general, there are two types of noise, i.e., additive noise and parametric noise. In the case of additive noise, noise is added to a certain deterministic equation, and in the case of parametric noise, a certain parameter of a deterministic equation is fluctuated by noise. Crutchfield et al. (1982) studied the effect of noise for logistic equation and showed that the effect of parametric noise is equivalent to that of additive noise. However, it is not always true for high dimensional system. In this study, we treat with the case of parametric noise because it is likely for the foreign exchange rate to be fluctuated by noise. For the details of numerical simulation of the Kaldorian business cycle model with parametric noise in a closed economy, see Dohtani et al. (1996). The following statement summarizes the results of our numerical simulation:

(1) The behavior of flexible foreign exchange rate $(E)$ begins to burst as the adjustment speed in the goods market $(\alpha)$ is increased in the system without noise.

(2) Noise may suppress the burst of exchange rate, in other words, noise may reveal the hidden structures.

(3) The windows of periodic solution can be broken by noise and the hidden chaotic structure may appear.

\subsection{The Appearing and the Suppressing of Burst}

Figure 1 is the bifurcation diagram of the foreign exchange rate $(E)$ without noise. The bifurcation parameter is the adjustment speed in the goods market $(\alpha)$. Figure 2 shows the largest Lyapunov exponent in this system. These figures indicate that the behavior of the exchange rate begins to burst and chaotic behaviors appear frequently when

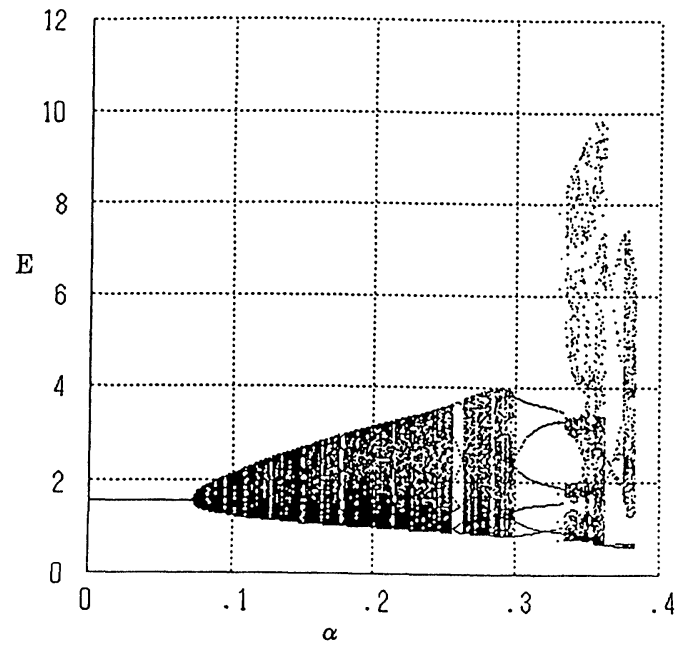

FIGURE 1 The bifurcation diagram of $E$ without noise.

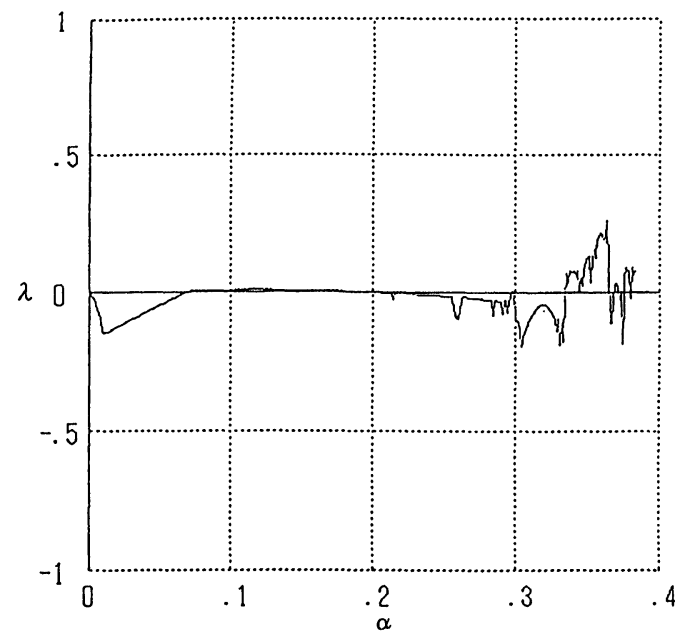

FIGURE 2 The largest Lyapunov exponent without noise.

$\alpha \geq 0.33$. Figure 3 is the chaotic attractor in the $E-Y$ plane without noise when $\alpha=0.33$.

However, noise can suppress the burst of the exchange rate. Figure 4 is the attractor which is revealed by the small noise $(\sigma=0.01)$ when $\alpha=0.33$. This attractor is similar to that in the case of $\alpha=0.32$, which is cyclical and not burst. From the economic point of view, this means that even if the foreign exchange rate fluctuates heavily when there is no stochastic disturbance, this heavy 


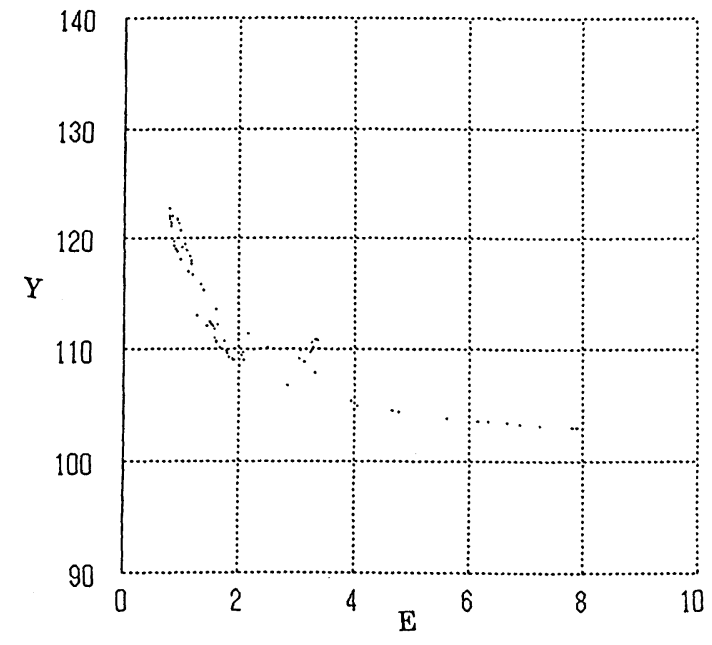

FIGURE 3 The attractor in $E-Y$ plane without noise $(\alpha=0.33)$

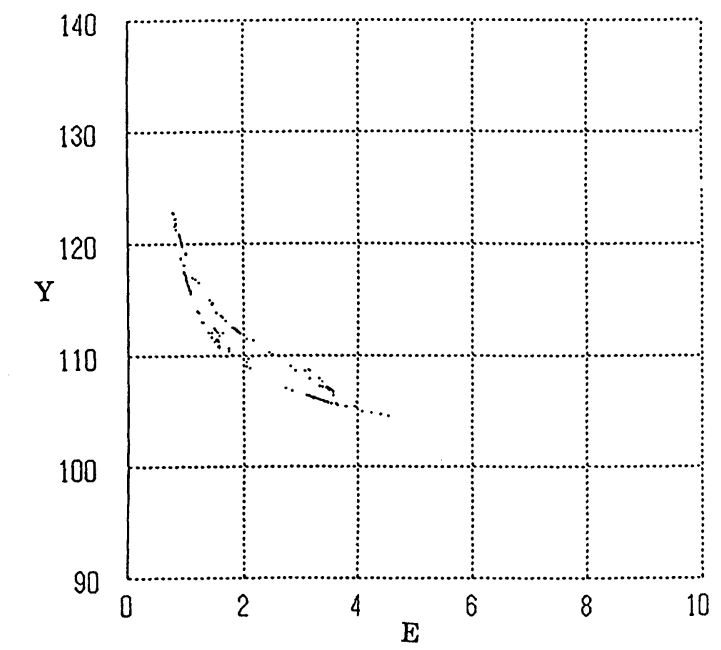

FIGURE 4 The attractor in $E-Y$ plane with noise $(\alpha=0.33, \sigma=0.01)$.

fluctuation may be suppressed by a small noise. Figures 5 and 6 compare the trajectory of the exchange rate when $\alpha=0.33$ without noise to that with small noise $(\sigma=0.01)$.

The above example shows that the noise may stabilize the system. But, this does not mean that the noise always stabilize the system. Figures 7 and 8 are two examples of the experiments with somewhat larger noise $(\sigma=0.05)$. These examples show that the noise can destabilize rather than stabilize the

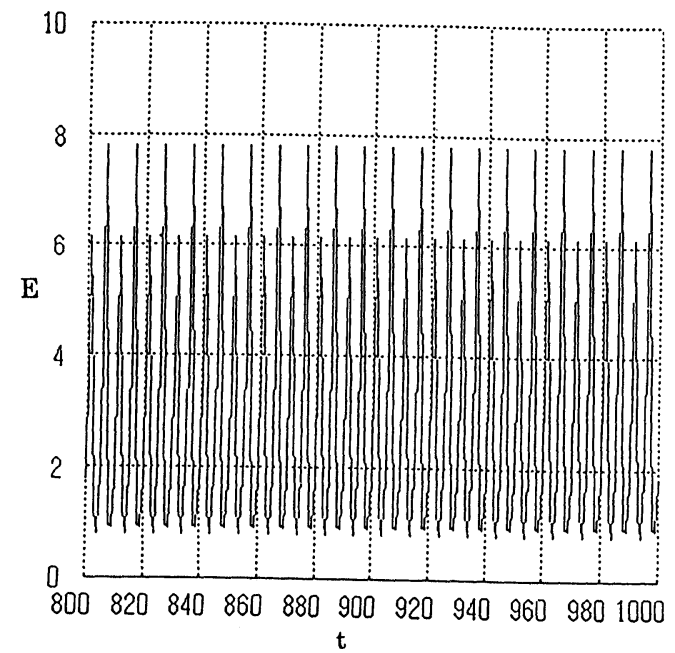

FIGURE 5 A trajectory of $E$ without noise $(\alpha=0.33)$.

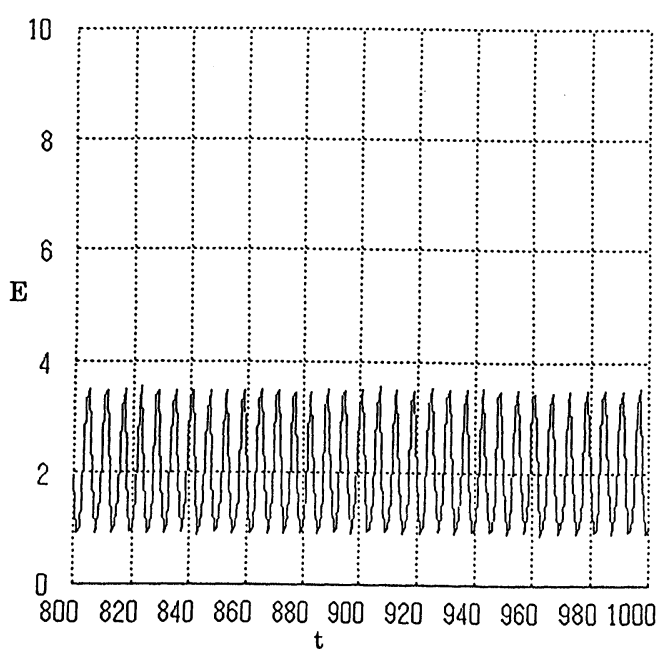

FIGURE $6 \quad$ A trajectory of $E$ with noise $(\alpha=0.33, \sigma=0.01)$.

system in some situations. Figure 9 is a revealed attractor in the $E-Y$ plane when $\alpha=0.33$ and $\sigma=0.05$, which is chaotic and similar to that for $\alpha=0.34$ without noise. To sum up, noise can reveal the hidden structures.

\subsection{The Broken Windows}

By comparing the largest Lyapunov exponent and bifurcation diagram without noise (Figs. 1 and 2) 


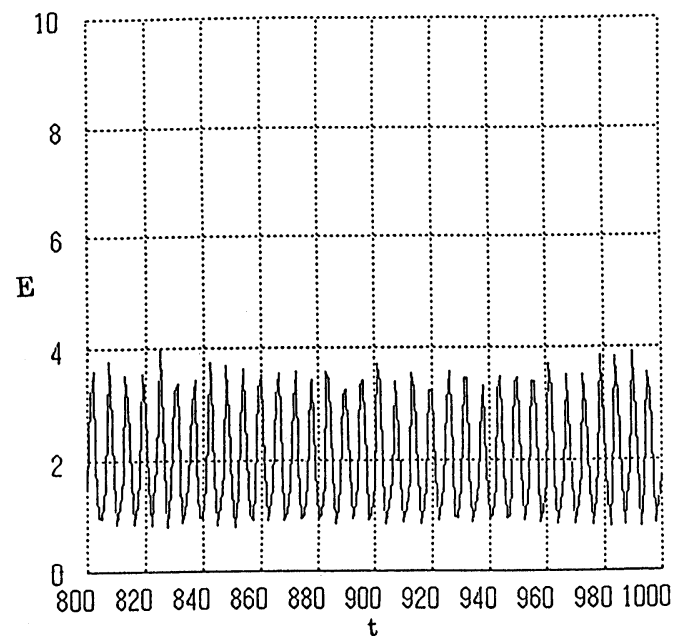

FIGURE 7 A trajectory of $E$ with noise $(\alpha=0.33, \sigma=0.05)$.

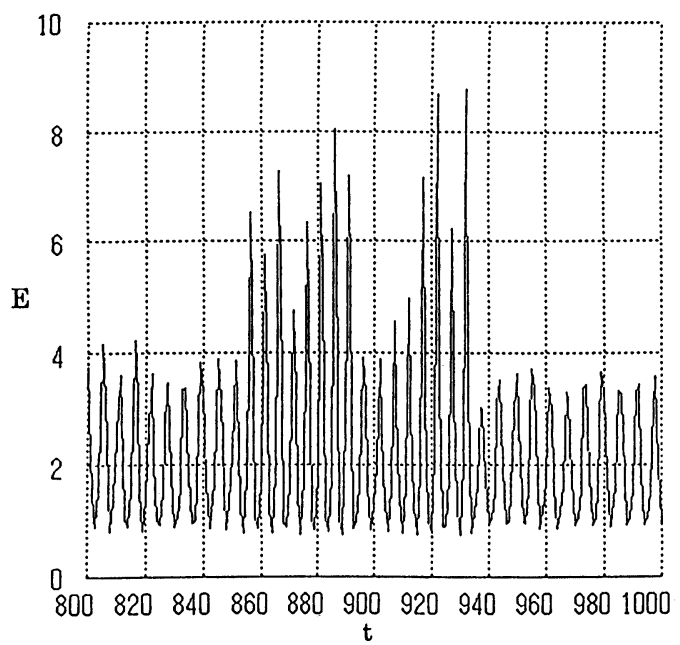

FIGURE $8 \quad$ A trajectory of $E$ with noise $(\alpha=0.33, \sigma=0.05)$.

with those with noise (Figs. 10 and 11), we can see that noise may obscure the windows of cycle for the exchange rate $(E)$. This is also true for the behavior of national income $(Y)$. This example shows that noise can reveal the hidden chaotic structure even if the behavior of the system without noise is periodic.

\section{CONCLUDING REMARKS}

In this paper, we studied the economic implications of the noise effects by using an analytical

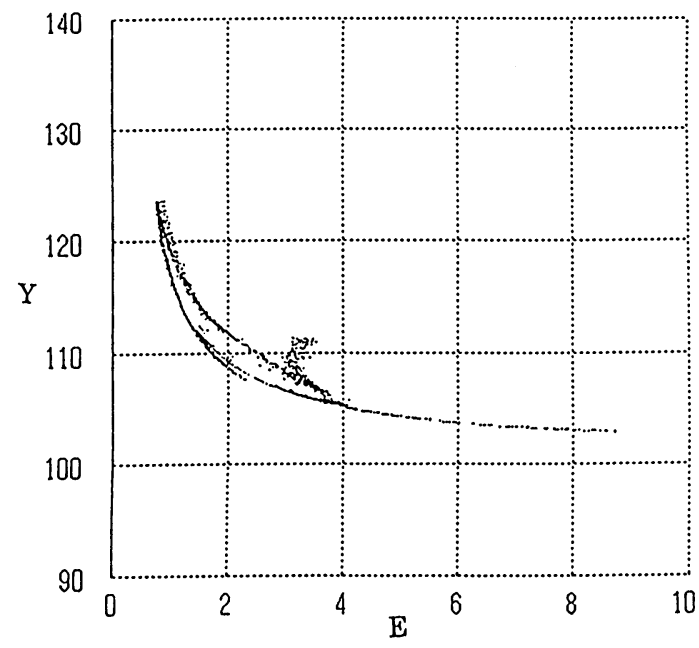

FIGURE 9 The attractor in $E-Y$ plane with noise $(\alpha=0.33, \sigma=0.05)$.

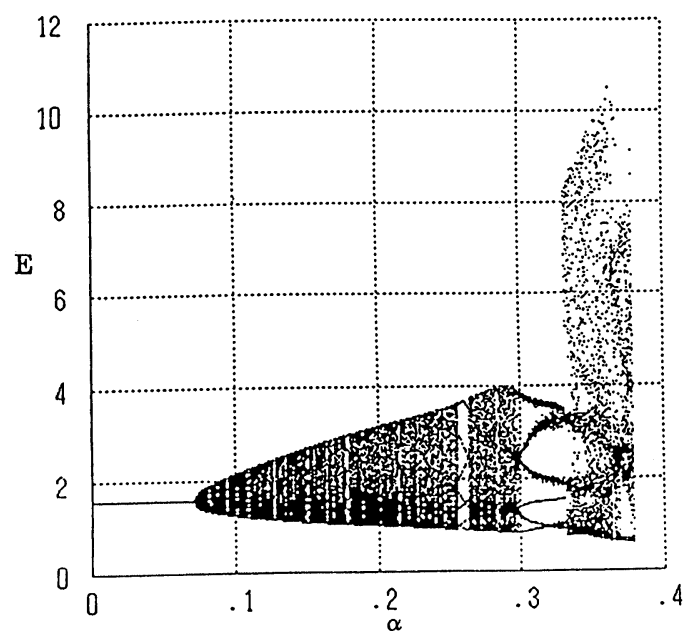

FIGURE 10 The bifurcation diagram of $E$ with noise.

framework of the discrete time version of Kaldorian business cycle model in a small open economy with flexible exchange rates. This is a good starting point to the study of the economic interactions between countries or regions. The effective range of the model of small open economy is, however, rather restricted, because many variables such as national income or rate of interest of foreign country are supposed to be given outside of the system. Multi-country or multi-regional model may be more appropriate for the study of the 


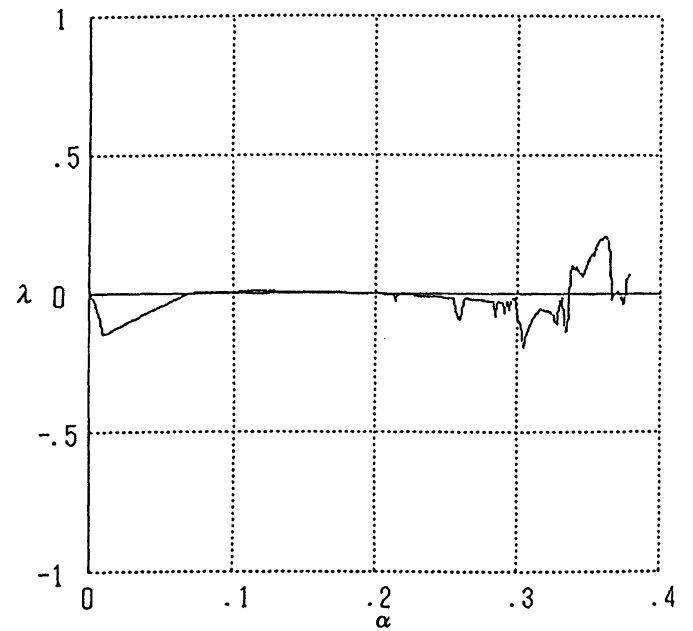

FIGURE 11 The largest Lyapunov exponent with noise.

dynamic interactions between regions. The simplest version of such a model is two country model. The analysis of such a complicated system is beyond the scope of the present paper and it is left for our research in future.

\section{Acknowledgement}

This paper was presented at PRSCO 16 (The 16th Pacific Regional Science Conference) which was held at Seoul, Korea (July 15, 1999). Thanks are due to the valuable comment by Prof. Tamotsu Onozaki of Asahikawa University. This work was financially supported by the Grant-in-Aid for Scientific Research No. 09640285 from the Ministry of Education, Science and Culture of Japanese Government, Grant-in-Aid for Scientific Research No. 11630020 by Japan Society for the Promotion of Science, and Waseda University Grant for Special Research Projects No. 99A-107.

\section{References}

Asada, T. (1995): Kaldorian dynamics in an open economy. Journal of Economics/Zeitschrift für Nationalökonomie $\mathbf{6 2}$, 239-269.

Asada, T., Inaba, T. and Misawa, T. (1998): A nonlinear macrodynamic model with fixed exchange rates: Its dynamics and noise effects. Paper presented at, The First International Conference on DCDNS (Discrete Chaotic Dynamics in Nature and Society), in Beer-Sheva, Israel (October 21, 1998).

Crutchfield, J.P., Farmer, J.D. and Huberman, B.A. (1982): Fluctuations and simple chaotic dynamics. Physics Reports $\mathbf{9 2}$, $45-82$.

Dohtani, A., Misawa, T., Inaba, T., Yokoo, M. and Owase, T. (1996): Chaos, complex transients and noise: Illustrations with a Kaldor model. Chaos, Solitons and Fractals 7, 2157-2174.

Gandolfo, G. (1996): Economic Dynamics (Third edn.) Berlin, Heidelberg, New York and Tokyo: Springer-Verlag.

Kaldor, N. (1940): A model of the trade cycle. Economic Journal 50, 78-92. 


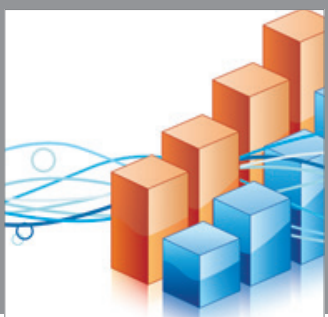

Advances in

Operations Research

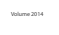

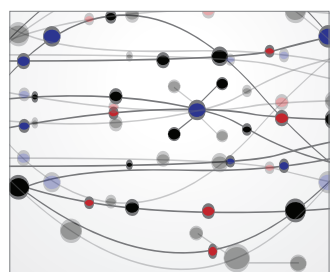

\section{The Scientific} World Journal
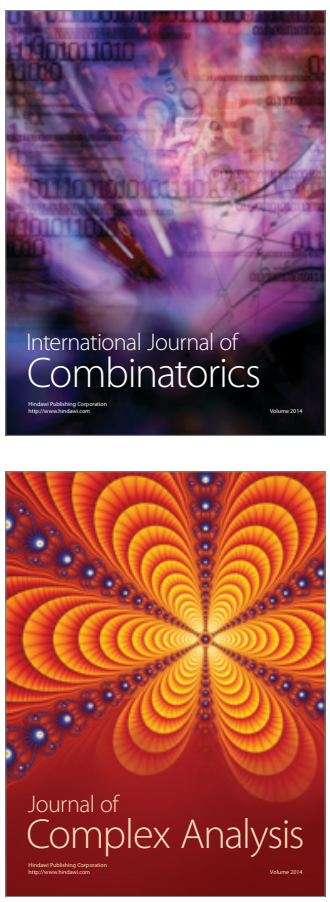

International Journal of

Mathematics and

Mathematical

Sciences
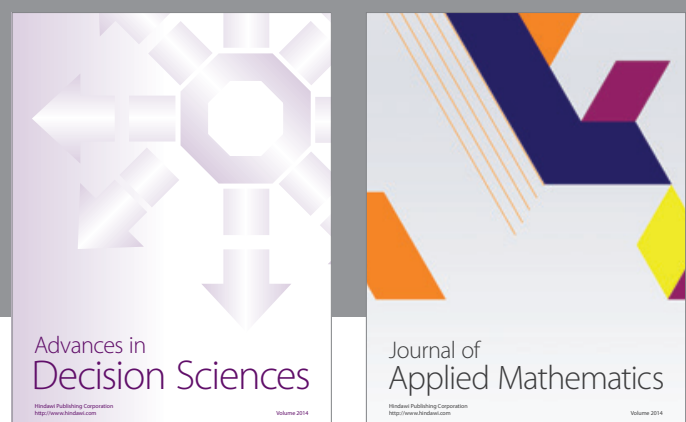

Journal of

Applied Mathematics
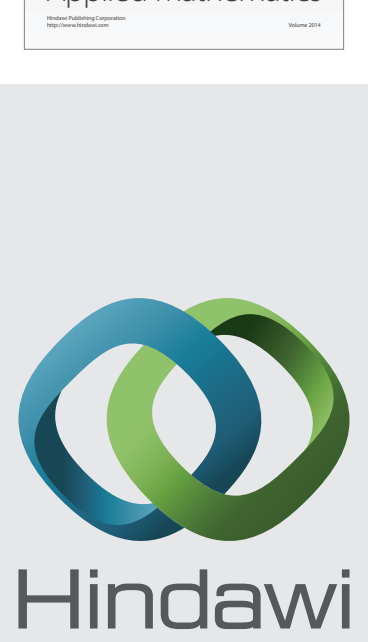

Submit your manuscripts at http://www.hindawi.com
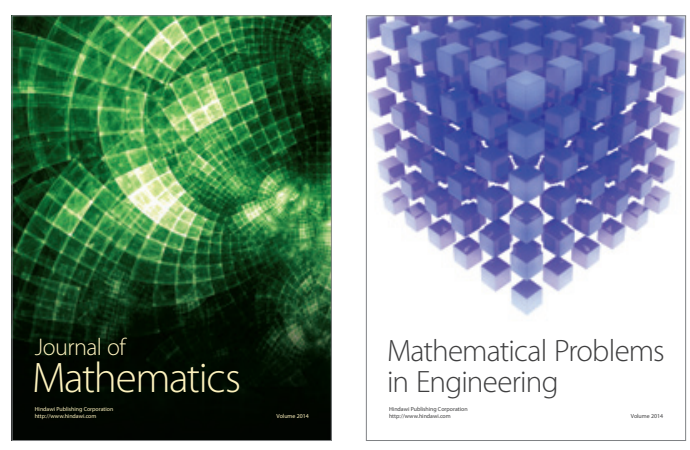

Mathematical Problems in Engineering
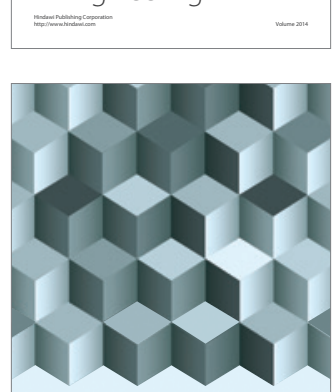

Journal of

Function Spaces
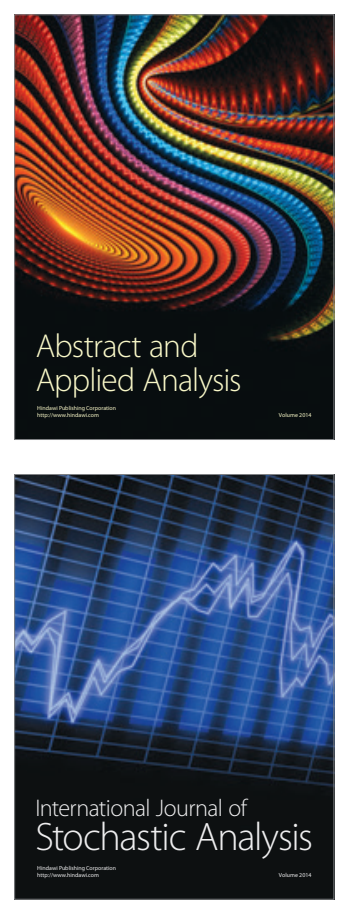

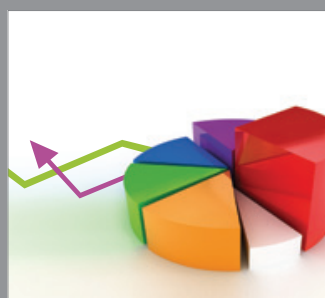

ournal of

Probability and Statistics

Promensencen
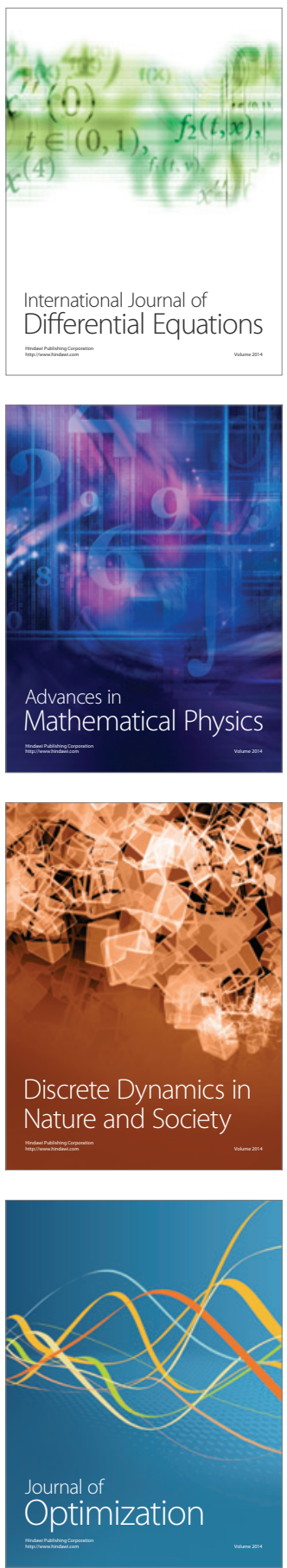